MATEC Web of Conferences 8, 01001 (2013)

DOI: $10.1051 /$ matecconf $/ 20130801001$

C Owned by the authors, published by EDP Sciences, 2013

\title{
Spatial chirp control of high-intensity 4D pulse focusing for laser-matter interactions
}

\author{
C. Durfee, M. Greco, A. Meier, E. Block, J. Squier \\ Center for Microintegrated Optics for Advanced Bioimaging and Control \\ Department of Physics \\ Colorado School of Mines \\ Golden, CO, USA 80401
}

\begin{abstract}
Spatial chirp can be manipulated to control the focusing conditions for materials processing. Our double-ABCD nonparaxial analysis helps to understand and exploit the mechanisms for intensity localization, pulse front tilt, and grating formation, and includes initial spectral phase and detuning of the wavelength crossing plane. We also present a novel method for creating high density, high intensity interference patterns with crossed beams that have no relative pulse front tilt.
\end{abstract}

\section{Non-paraxial double ABCD tracing}

High-intensity laser-matter interactions inside a medium are complicated by nonlinear propagation along the way to the focal spot. Self-focusing, ionization defocusing and filamentation can prevent an ultrashort pulse from reaching its highest intensity. While focusing with high numerical aperture can suppress nonlinear propagation in advance of the focal plane, the area of the spot is necessarily on the scale of the wavelength. Control of the spatial distribution of the frequency components of an ultrafast pulse can lead to an axial localization of the intensity that is much smaller than the confocal parameter for the focal spot[1,2]. The technique of focusing spatially-chirped beams suppresses the intensity away from the focal plane, enabling our demonstration of micromachining on the backside of transparent materials[3,4]. Since the pulse duration gets shorter as the beam comes to a focus and the intensity is strongly localized in the axial direction, the pulse is effectively focused in 4D.

The properties of space-time focused beams are strongly dependent of the nature of the spatial overlap of the frequency components. The analysis of optical systems with spatial chirp is therefore critical both for understanding the unusual properties of these beams and for designing systems to exploit these properties for materials processing. In a recent paper, we developed an analytical approach to calculate the propagation of spatially chirped beams. A ray is traced that represents the central axis of the Gaussian beamlet for each frequency component. Since one form of the calculation is nonparaxial, the beamlet angles can be large and the calculation is valid over a wider range of conditions than direct Fresnel propagation. Our technique explains, for example, how the evolution of the beamlet radius of curvature leads to an axial variation of the spectral chirp through the focus, contributing to the axial intensity localization. It also allows the derivation of simple scaling properties of the beam, showing that the improvement of the localization over the confocal parameter of the focused beamlets depends primarily on the degree of spatial chirp, not on the initial pulse duration or the focal length.
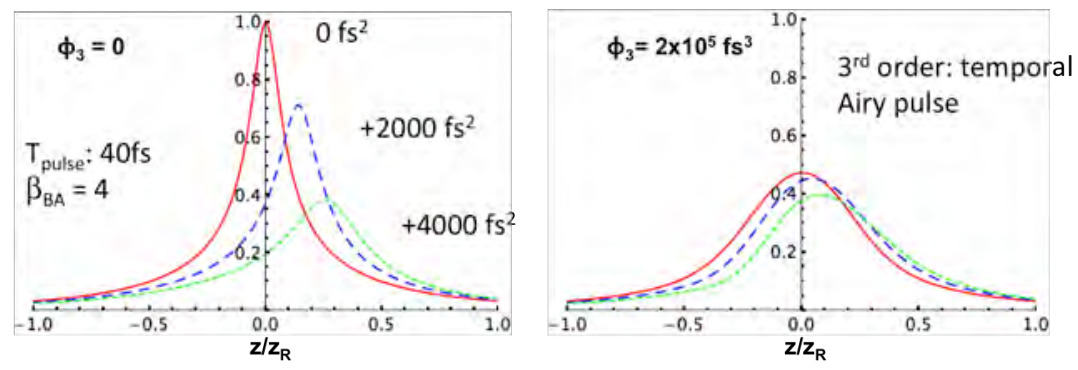

Figure 1. Axial intensity profiles for an SSTF pulse as the initial second-order spectral phase is varied. Left: Zero initial thirdorder phase. Right: Positive third-order phase.

\section{General detuning of spatial chirped beams}


Although space-time focusing is fairly robust to misalignment, the best localization will take place only when the optical alignment is optimized. Figure 1 shows how the axial intensity varies with input spectral chirp in the presence of zero and a large degree of third-order chirp. In the first case, the position of the shortest pulse translates as the input chirp adjustment combines with the geometric spectral chirp mentioned above. With strong third-order phase, the shape of the pulse is not as sensitive to input chirp, so the axial peak intensity does not translate as much. This is a scenario that can easily arise when the incident angle of the compressor of the laser system or the SSTF system is imperfectly aligned. We can also use the double ABCD formulation to calculate the field for the case where the planes for the beamlet waists and the wavelength crossing are different. This situation occurs when the beamlet divergence is mismatched to the geometric focal plane of the lens. The results is that there are two distinct focal planes, one where the beamlets are focused but where there is a transverse spatial chirp, and another where the pulse duration is shortest but the beamlets have some divergence. We find that for machining the SSTF for a detuned set up still results in better intensity localization than for conventional focusing, but to achieve the full localization effects, careful alignment and characterization is necessary.

\section{High intensity pulse front matched femtosecond grating formation}

Interfering laser beams are used to create grating structures in materials, but crossing ultrshort pulses leads to a time sweep across the focal spot owing to the mismatch in pulse fronts. In early work, Maznev et al showed that femtosecond pulses can be made to overlap by using $4 \mathrm{f}$ imaging of the $+/-1$ orders from a diffraction grating[5]. In the imaging process, the pulse fronts approach the crossing point so that there is even temporal overlap across the whole focal spot. This pulse front matching works only for 1:1 magnification, so it cannot be used to produce a higher intensity on target than at the grating. We have developed a novel arrangement in which a second grating is used in place of the first lens to collimate the beamlets from the first grating. Provided the grating separation is equal to the focal length of the focusing optics, the pulse fronts will be matched. Unlike the $4 \mathrm{f}$ system, the beamlets can now be focused tightly to produce a high-intensity interference with pulse front matching.
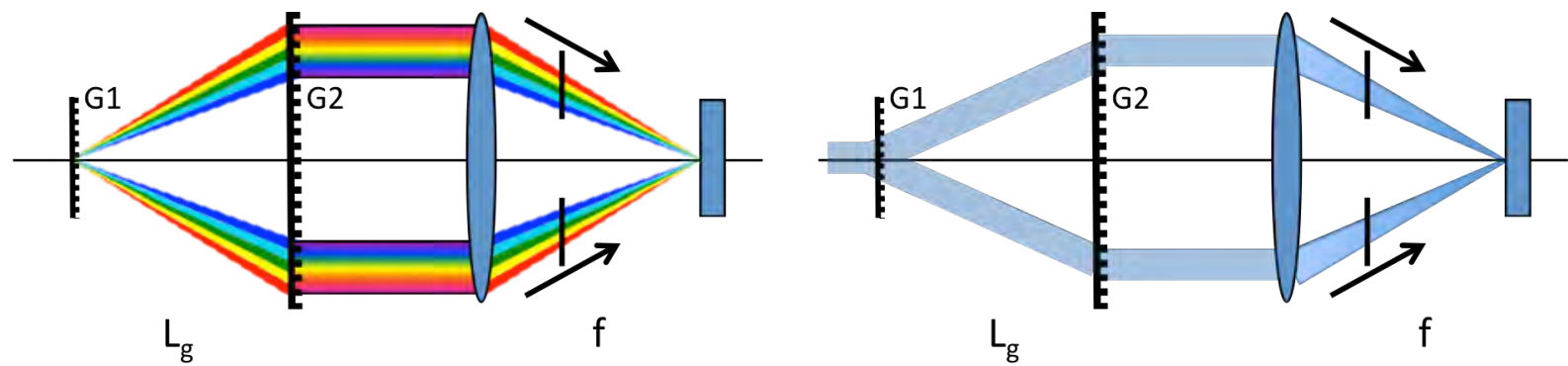

Figure 2. Optical arrangement for the production of high-intensity pulse front matched interference. The pulse fronts are matched when the grating separation $L_{g}$ is equal the lens focal length $f$.

\section{Acknowledgments}

This work was supported by The US Air Force Office of Scientific Research under programs FA9550-10-0561 and FA9550-12-1-0482.

\section{References}

1. G. Zhu, J. van Howe, M. Durst, W. Zipfel, and C. Xu, "Simultaneous spatial and temporal focusing of femtosecond pulses," Opt Express 13, 2153-2159 (2005).

2. C. G. Durfee, M. Greco, E. Block, D. Vitek, and J. A. Squier, "Intuitive analysis of space-time focusing with doubleABCD calculation," Opt Express 20, 14244 (2012).

3. D. N. Vitek, D. E. Adams, A. Johnson, P. S. Tsai, S. Backus, C. G. Durfee, D. Kleinfeld, and J. A. Squier, "Temporally focused femtosecond laser pulses for low numerical aperture micromachining through optically transparent materials," Opt Express 18, 18086-18094 (2010).

4. D. N. Vitek, E. Block, Y. Bellouard, D. E. Adams, S. Backus, D. Kleinfeld, C. G. Durfee, and J. A. Squier, "Spatiotemporally focused femtosecond laser pulses for nonreciprocal writing in optically transparent materials," Opt Express 18 , 24673-24678 (2010).

5. A. Maznev, T. Crimmins, and K. Nelson, "How to make femtosecond pulses overlap," Opt Lett 23, 1378-1380 (1998). 